\title{
Analysis of Frequency Bands of Uterine Electromyography Signals for the Detection of Preterm Birth
}

\author{
Vinothini SELVARAJU ${ }^{\mathrm{a}, 1}$, P.A KARTHICK ${ }^{\mathrm{b}}$ and Ramakrishnan SWAMINATHAN ${ }^{\mathrm{a}}$ \\ ${ }^{a}$ Biomedical Engineering Group, Department of Applied Mechanics, Indian Institute of \\ Technology Madras, Tamilnadu, India \\ ${ }^{\mathrm{b}}$ Department of Instrumentation and Control Engineering, National Institute of \\ Technology Tiruchirappalli, Tamilnadu, India
}

\begin{abstract}
In this work, an attempt has been made to analyze the influence of the frequencies bands in uterine electromyography (uEMG) signals on the detection of preterm birth. The signals recorded from the women's abdomen during pregnancy are considered in this study. The signals are subjected to preprocessing using digital bandpass Butterworth filter and decomposed into different frequency bands namely, 0.3-1.0 Hz (F1), 1.0-2.0 Hz (F2) and 2.0-3.0Hz (F3). Spectral features namely, peak magnitude, peak frequency, mean frequency and median frequency are extracted from the power spectrum. Classification models namely, k-nearest neighbor, support vector machine and random forest are employed to distinguish the term and preterm conditions. The results show that the features extracted from these frequency bands are able to differentiate term and preterm condition. Particularly, the frequency band F3 performs better than other frequency bands. The features associated with these frequencies along with random forest classification model achieves a maximum accuracy of $75.2 \%$. Thus, these measures could be used to accurately detect the preterm birth well in advance.
\end{abstract}

Keywords. Term, Preterm, Spectral features, uterine Electromyography

\section{Introduction}

During pregnancy, women undergo significant anatomical and physiological changes in order to nourish the growing fetus [1]. Despite being a natural process, it involves health complications leading to the fetus and mother's morbidity and mortality [2]. Every year approximately 15 million neonates are born preterm, accounting for $11 \%$ of the global birth rate which presents a public health concern [3]. Preterm infants suffer from various neurodevelopment impairments and disabilities [2]. Therefore, monitoring the physiological changes in the pregnant women and fetus is important in the current scenario.

Uterine Electromyography (uEMG) is a non-invasive method of measuring uterine activity from the abdominal surface. It monitors the frequency, duration and strength of

\footnotetext{
${ }^{1}$ Corresponding Author, Vinothini Selvaraju, Biomedical Engineering Group, Department of Applied Mechanics, Indian Institute of Technology Madras, Chennai, India; E-mail: vinothiniiitm@gmail.com.
} 
uterine contractions [4]. The analysis of $\mathrm{uEMG}$ signals can provide information about the onset of labor and predict preterm birth.

Several researches have been explored on the uEMG signals to detect preterm birth. Time domain features such as, root mean square, integrated EMG, and log detector have been used [6][7]. Similarly, spectral features such as, peak frequency, median frequency and mean frequency have also been explored. Most of the studies analyzed uterine burst signals in the frequency range of $0.3-1 \mathrm{~Hz}$ [2]. However, few attempts have been made to analyze the different frequency ranges of $\mathrm{uEMG}$ signals [8].

In this work, an attempt has been made to analyse the different frequency bands of uEMG signals from three different channels. Spectral features associated with the frequency components of signals are extracted and classifiers are employed to discriminate term and preterm conditions.

\section{Methods}

\subsection{Database description}

The uEMG signals considered from the Physionet Term-Preterm ElectroHysteroGram dataset are used in this study [6]. It consists of $300 \mathrm{uEMG}$ signals that are recorded by the bipolar electrodes from the abdominal surface. Four electrodes are placed around the navel with an interelectrode distance of $7 \mathrm{~cm}$. Further details on the positioning of the electrodes and channels have been explained in [6]. The recording duration and sampling frequency is 30 minutes and $20 \mathrm{~Hz}$ respectively. These signals are pre-processed using Butterworth band-pass filters with various cut-off frequencies namely, 0.08-4 Hz, 0.34.0 Hz and $0.3-3.0 \mathrm{~Hz} .143$ term signals and 19 preterm signals recorded prior to 26 weeks of gestation at the frequency range of $0.3-3 \mathrm{~Hz}$ are considered in the study.

\subsection{Preprocessing}

The spectral characteristics of the UEMG signal are divided into a specific sub-band. These sub-bands used in this study are $0.3-1.0 \mathrm{~Hz}(\mathrm{~F} 1), 1.0-2.0 \mathrm{~Hz}$ (F2) and $2.0-3.0$ $\mathrm{Hz}$ (F3) which focus on the energy distribution in the different frequency bands. These frequency bands are filtered using Butterworth bandpass filters [8].

\subsection{Features extraction}

Four spectral features are extracted from the decomposed sub-bands and description is given below:

- Peak Magnitude (PM) - It is the occurrence of the maximum power in the spectrum [8].

- Peak frequency $(P K F)$ - PKF is the frequency that corresponds to maximum power in the spectrum [6].

- Mean frequency (MNF) - MNF is the frequency that is calculated as a ratio of the total sum of product of the signal frequencies with its power spectrum to the sum of the intensity of the spectrum [9]. It is given as, 


$$
\text { Mean frequency }=\sum_{\mathrm{i}=1}^{\mathrm{M}} \mathrm{f}_{\mathrm{i}} \mathrm{P}_{\mathrm{i}} / \sum_{\mathrm{i}=1}^{\mathrm{M}} \mathrm{P}_{\mathrm{i}}
$$

where, $P_{i}$ is the power spectrum of the uEMG signal, and $f_{i}$ is the frequency in the spectrum.

- Median frequency $(M D F)$ - MDF is the intermediate frequency in which the power spectrum is separated into two parts equally [6]. It is mathematically written as,

$$
\sum_{i=1}^{\mathrm{MDF}} \mathrm{P}_{\mathrm{i}}=\sum_{\mathrm{i}=\mathrm{MDF}}^{\mathrm{M}} \mathrm{P}_{\mathrm{i}}
$$

\subsection{Data-balancing}

The number of records in the term and preterm conditions are not balanced. It has an impact on the statistical analysis and performance of classification model's performance and are prone to detect the majority class (term). The Adaptive synthetic sampling method (ADASYN) is employed on the preterm signals to increase the minority class samples [10]. In this study, 10-fold cross validation technique is employed. To obtain the unbiased stable classification model, the training data is only oversampled using ADASYN [11].

\subsection{Statistical analysis and classifiers}

The Anderson-Darling test is utilized to examine the normality of the data and Wilcoxon's rank sum test is performed to analyze the statistical significance. Three classifiers namely, k-nearest neighbor $(\mathrm{KNN})$, support vector machine (SVM) and random forest (RF) are considered [8]. The performance of the classifier is analyzed using metrics namely, accuracy (Acc), F-measure (F) and area under the curve (AUC).

\section{Results and discussion}

The representative uEMG signal is shown in Figure 1 for different channels. The signal amplitude of channel 1, 2 and 3 for the term condition as seen in Figure 1 (a) varies from $-54.3 \mu \mathrm{V}$ to $44.7 \mu \mathrm{V},-37.3 \mu \mathrm{V}$ to $26.1 \mu \mathrm{V}$, and $-21.5 \mu \mathrm{V}$ to $21.5 \mu \mathrm{V}$ respectively. The amplitude of the signal in channel 1, 2 and 3 for the preterm condition as observed from in Figure 1 (b) lies from $-34.9 \mu \mathrm{V}$ to $33.2 \mu \mathrm{V},-60.3 \mu \mathrm{V}$ to $57.3 \mu \mathrm{V}$, and $-32.0 \mu \mathrm{V}$ to 28.6 $\mu \mathrm{V}$. Hence, the signal amplitude of the preterm condition is observed to be higher with more spikes than the term condition.

The power spectrum of term and preterm condition is shown in Figure 2. It is seen that the power of the signals is observed to vary across the channel and lie within the frequency of $2 \mathrm{~Hz}$. Two peaks are seen in the term condition [6]. It is observed that peak corresponding to lower frequencies is found to be high in the term condition compared to preterm condition. It is also found that high frequency peak clearly seen in Figure 2 (a). This may be attributed to the reduction in the bandwidth for preterm conditions. The statistical significance of the four spectral features for channel 1, channel 2 and channel 3 with different frequency bands are calculated. It is found that peak magnitude in 
channel 3 is observed to have significant difference in differentiating term and preterm conditions [6].

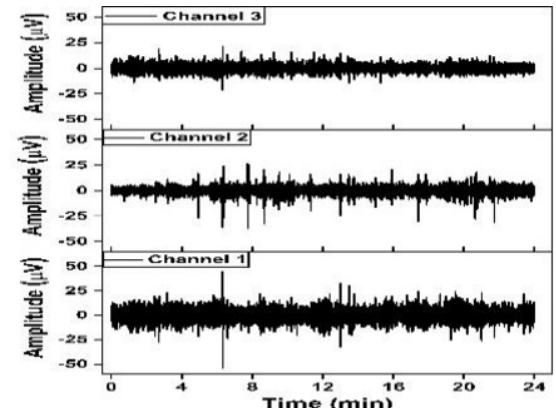

(a)

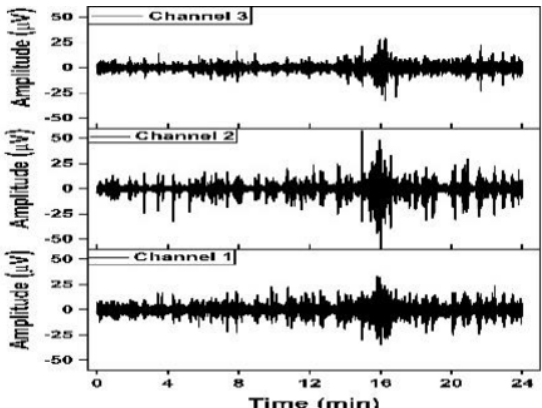

(b)

Figure 1. Representative uEMG signals in a) Term and b) Preterm condition for different channels

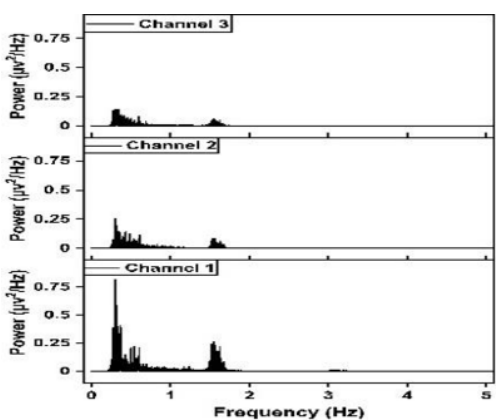

(a)

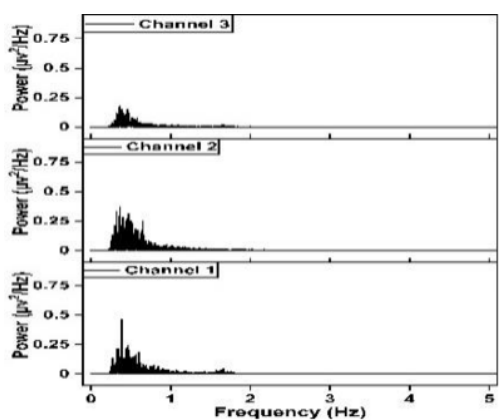

(b)

Figure 2. Power spectrum of a) Term and b) Preterm condition shown in Figure 1.

Table 1 Classification performance of different frequency bands

\begin{tabular}{cc|ccc|ccc|ccc}
\hline \multirow{2}{*}{ Channel } & \multirow{2}{*}{ Classifiers } & \multicolumn{3}{|c|}{ F1 } & \multicolumn{3}{|c|}{ F2 } & \multicolumn{3}{c}{ F3 } \\
\cline { 3 - 10 } & & Acc & F & AUC & Acc & F & AUC & Acc & F & AUC \\
\hline \multirow{2}{*}{1} & KNN & 59.9 & $\mathbf{3 0 . 3}$ & 45.7 & 64.7 & $\mathbf{4 3 . 1}$ & 57.1 & 64.0 & 29.3 & 52.9 \\
& SVM & 33.0 & 21.3 & $\mathbf{4 7 . 1}$ & 55.0 & 30.8 & 51.7 & 55.5 & $\mathbf{3 1 . 2}$ & $\mathbf{6 9 . 0}$ \\
& RF & $\mathbf{6 6 . 7}$ & 27.7 & 34.5 & $\mathbf{7 4 . 0}$ & 33.3 & $\mathbf{5 8 . 2}$ & $\mathbf{7 1 . 0}$ & 30.0 & 63.4 \\
& & & & & & & & & & \\
2 & KNN & 64.2 & 35.2 & 49.2 & 67.3 & 28.7 & 62.2 & 54.4 & 27.0 & 52.2 \\
& SVM & 37.6 & 23.7 & 51.7 & 64.2 & 39.7 & $\mathbf{7 5 . 9}$ & 48.1 & 27.0 & 55.6 \\
& RF & $\mathbf{7 1 . 6}$ & $\mathbf{3 6 . 5}$ & $\mathbf{5 2 . 9}$ & $\mathbf{7 4 . 6}$ & $\mathbf{3 9 . 9}$ & 66.3 & $\mathbf{7 5 . 2}$ & $\mathbf{3 4 . 6}$ & $\mathbf{6 7 . 7}$ \\
& & & & & & & & & & \\
3 & KNN & 62.4 & 36.7 & 43.2 & 69.0 & 34.2 & 58.0 & 58.7 & 33.3 & 37.8 \\
& SVM & 53.6 & 28.1 & $\mathbf{5 8 . 9}$ & 53.1 & 24.7 & 53.6 & 55.0 & 29.3 & $\mathbf{6 7 . 0}$ \\
& RF & $\mathbf{7 0 . 4}$ & $\mathbf{3 8 . 1}$ & 51.7 & $\mathbf{7 4 . 6}$ & $\mathbf{3 7 . 4}$ & $\mathbf{6 3 . 5}$ & $\mathbf{6 7 . 9}$ & $\mathbf{4 0 . 0}$ & 45.2 \\
\hline
\end{tabular}

The performance of the spectral features is analyzed with machine learning models for different frequency bands and are presented in Table 1. Channel 1, 2 and 3 provide an accuracy of $74.0 \%, 75.2 \%$ and $74.6 \%$ respectively with RF classifier. Further, RF classifier is found to result in accuracy of $71.6 \%, 74.6 \%$ and $75.2 \%$ in frequency bands $\mathrm{F} 1, \mathrm{~F} 2$ and F3 respectively. It is evident from these results that channel 2 frequency band F3 is optimal for classification of term and preterm condition. However, the limited dataset cannot be used effectively to develop the machine learning model and, in the future, data will be recorded to develop better models. 


\section{Conclusions}

In this work, uEMG signals considered prior to 26 weeks of gestation are analyzed using spectral features for different frequency bands and three different channels. From the preprocessed signals, uEMG signals are filtered into different frequency bands using Butterworth band-pass filter. Spectral features are extracted from uEMG signals for the term and preterm condition. Class imbalance problem is eliminated using oversampling method. These term and balanced preterm features are given as the input to the machine learning models namely, KNN, SVM and RF. The spectral features in the frequency band of F3 are found to have better performance than others. The RF model combined with spectral features achieves a maximum accuracy in differentiating the term and preterm conditions. However, further investigations based on nonlinear features are needed due to the complex physiological mechanism of uterus and will be explored in near future.

\section{References}

[1] Soma-Pillay P, Catherine NP, Tolppanen H, Mebazaa A, Tolppanen H, Mebazaa A. Physiological changes in pregnancy. CVJA. 2016 Mar;27(2):89.

[2] Garcia-Casado J, Ye-Lin Y, Prats-Boluda G, Mas-Cabo J, Alberola-Rubio J, Perales A. Electrohysterography in the diagnosis of preterm birth: a review. Physiol meas. 2018 Feb 26;39(2):02TR01.

[3] Walani SR. Global burden of preterm birth. Int J Gynecol Obstet. 2020 Jul;150(1):31-3.

[4] Lucovnik M, Maner WL, Chambliss LR, Blumrick R, Balducci J, Novak-Antolic Z, Garfield RE. Noninvasive uterine electromyography for prediction of preterm delivery. Am J obstet Gynecol. 2011 Mar 1;204(3):228-e1.

[5] Punitha N, Ramakrishnan S. Multifractal analysis of uterine electromyography signals to differentiate term and preterm conditions. Proc Inst Mech Eng H. 2019 Mar;233(3):362-71.

[6] Fele-Žorž G, Kavšek G, Novak-Antolič Ž, Jager F. A comparison of various linear and non-linear signal processing techniques to separate uterine EMG records of term and pre-term delivery groups. MBEC. 2008 Sep 1;46(9):911-22.

[7] Hemthanon C, Janjarasjitt S. Examination of Time-Domain Features of EHG Data for Preterm-Term Birth Classification. J Comput. 2019;30(2):41-54.

[8] Jager F, Libenšek S, Geršak K. Characterization and automatic classification of preterm and term uterine records. PLoS One. 2018 Aug 28;13(8):e0202125.

[9] Alberola-Rubio J, Garcia-Casado J, Prats-Boluda G, Ye-Lin Y, Desantes D, Valero J, Perales A. Prediction of labor onset type: Spontaneous vs induced; role of electrohysterography?. Comput Meth Prog Bio. 2017 Jun 1;144:127-33.

[10] Acharya UR, Sudarshan VK, Rong SQ, Tan Z, Lim CM, Koh JE, Nayak S, Bhandary SV. Automated detection of premature delivery using empirical mode and wavelet packet decomposition techniques with uterine electromyogram signals. Comput biol med. 2017 Jun 1;85:33-42.

[11] Vandewiele G, Dehaene I, Kovács G, Sterckx L, Janssens O, Ongenae F, De Backere F, De Turck F, Roelens K, Decruyenaere J, Van Hoecke S. Overly optimistic prediction results on imbalanced data: a case study of flaws and benefits when applying over-sampling. Arti Intell Med. 2021 Jan 1;111:101987. 